\title{
Natural Deep Eutectic Solvents for the Extraction of Bioactive Steroidal Saponins from Dioscoreae Nipponicae Rhizoma
}

\author{
Gui-Ya Yang ${ }^{1,+}{ }^{\dagger}$ Jun-Na Song ${ }^{1,+}$, Ya-Qing Chang ${ }^{1}$, Lei Wang ${ }^{1}$, Yu-Guang Zheng ${ }^{1,2}$, Dan Zhang ${ }^{1, *}$ and \\ Long Guo $1, * \mathbb{C}$
}

1 Traditional Chinese Medicine Processing Technology Innovation Center of Hebei Province, Hebei University of Chinese Medicine, Shijiazhuang 050200, China; Yangguiya_310@163.com (G.-Y.Y.); junnassong@163.com (J.-N.S.); Czuoya_123@163.com (Y.-Q.C.); wanglei1031@126.com (L.W.); zyg314@163.com (Y.-G.Z.)

2 Hebei Chemical \& Pharmaceutical College, Shijiazhuang 050200, China

* Correspondence: zhangdan@hebcm.edu.cn (D.Z.); guolong@hebcm.edu.cn (L.G.); Tel.: +86-0311-8992-6017 (D.Z. \& L.G.)

+ These authors contributed equally to this work.

check for updates

Citation: Yang, G.-Y.; Song, J.-N.; Chang, Y.-Q.; Wang, L.; Zheng, Y.-G.; Zhang, D.; Guo, L. Natural Deep Eutectic Solvents for the Extraction of Bioactive Steroidal Saponins from Dioscoreae Nipponicae Rhizoma. Molecules 2021, 26, 2079. https:// doi.org/10.3390/molecules26072079

Academic Editors: Alexander Shikov and Ivana Radojcic Redovnikovic

Received: 3 March 2021

Accepted: 2 April 2021

Published: 5 April 2021

Publisher's Note: MDPI stays neutral with regard to jurisdictional claims in published maps and institutional affiliations.

Copyright: (c) 2021 by the authors. Licensee MDPI, Basel, Switzerland. This article is an open access article distributed under the terms and conditions of the Creative Commons Attribution (CC BY) license (https:/ / creativecommons.org/licenses/by/ $4.0 /)$.

\begin{abstract}
In the present study, a simple and environmentally friendly extraction method based on natural deep eutectic solvents (NADESs) was established to extract four bioactive steroidal saponins from Dioscoreae Nipponicae Rhizoma (DNR). A total of twenty-one types of choline chloride, betaine, and L-proline based NADESs were tailored, and the NADES composed of 1:1 molar ratio of choline chloride and malonic acid showed the best extraction efficiency for the four steroidal saponins compared with other NADESs. Then, the extraction parameters for extraction of steroidal saponins by selected tailor-made NADES were optimized using response surface methodology and the optimal extraction conditions are extraction time, $23.5 \mathrm{~min}$; liquid-solid ratio, $57.5 \mathrm{~mL} / \mathrm{g}$; and water content, $54 \%$. The microstructure of the DNR powder before and after ultrasonic extraction by conventional solvents (water and methanol) and the selected NADES were observed using field emission scanning electron microscope. In addition, the four steroidal saponins were recovered from NADESs by D101 macroporous resin with a satisfactory recovery yield between $67.27 \%$ and $79.90 \%$. The present research demonstrates that NADESs are a suitable green media for the extraction of the bioactive steroidal saponins from DNR, and have a great potential as possible alternatives to organic solvents for efficiently extracting bioactive compounds from natural products.
\end{abstract}

Keywords: natural deep eutectic solvents; Dioscoreae Nipponicae Rhizoma; steroidal saponins; response surface methodology; extraction

\section{Introduction}

Dioscoreae Nipponicae Rhizoma (DNR), the dried rhizome of Dioscorea nipponica Makino, is widely distributed in China, South Korea, and Russia, and has been used as a traditional herbal medicine for relieving cough and asthma, eliminating rheumatic aches, alleviating pain, and improving blood circulation [1]. Modern pharmacological studies have discovered that DNR possesses anti-tumor, anti-inflammatory, anti-diuretic, analgesic, anti-tussive, immunoregulatory, and cardiovascular properties [2-6]. Phytochemical studies disclosed that steroidal saponins, such as protodioscin, protogracillin, pseudoprotodioscin, and pseudoprotogracillin are the major bioactive constituents in DNR, which are mainly responsible for most of the pharmacological effects of this herbal medicine $[7,8]$. Therefore, the content of the bioactive steroidal saponins is an important index for the quality control and clinical practice of DNR. Several analytical methods, including thin layer chromatography, high performance liquid chromatography, and high performance liquid chromatography coupled with mass spectrometry, have been used for qualitative and quantitate analysis of bioactive steroidal saponins in DNR $[9,10]$. However, research focused on the extraction method for the bioactive steroidal saponins from DNR are still limited. 
Conventional organic solvents, such as methanol, ethyl acetate, acetone, and chloroform, are commonly used in the extraction of bioactive components from natural sources. However, the consumption of large amounts of these volatile and hazardous organic solvents may contribute to environmental pollution and leave unacceptable solvent residues in extracts. Green extraction methods which are environmentally friendly and sustainable for sample preparation have received more and more attention in past years [11].

Since being introduced as a new type of green solvents, deep eutectic solvents (DESs) have rapidly gained great interest as sustainable alternatives to hazardous organic solvents [12]. DESs are composed of a mixture consisting of hydrogen bond acceptors (HBAs) with hydrogen bond donors (HBDs), which are able to form intermolecular hydrogen bonds and van der Waals interactions. Owing to their superior properties, such as negligible volatility, non-flammability, adjustable viscosity, cost-effectiveness, and facile production processes, DESs have been extensively used in many different fields such as pharmaceuticals, catalysis, electrochemistry, and extractions [13-17]. When DESs were prepared by mixing two or more naturally occurring and biodegradable components, they were called natural deep eutectic solvents (NADESs). The availability, low cost, biodegradability, and environmental friendliness of the components makes the NADESs versatile alternatives to conventional organic solvents [18]. Recently, several studied have used NADESs for extraction and separation of different types of bioactive compounds, such as phenolic acids, flavonoids, and alkaloids from various plant materials [19-21]. Nonetheless, the number of reports on the application of NADESs for the extraction of bioactive compounds is still limited, and the efficiency of NADESs on extraction of bioactive steroidal saponins from DNR still remains unknown.

In the present study, in order to evaluate NADESs for the extraction of steroidal saponins, a series of choline chloride $(\mathrm{ChCl})$, betaine (Bet), and L-proline (Pro) based NADESs were tailored for ultrasonic extraction of four bioactive steroidal saponins, including protodioscin, protogracillin, pseudoprotodioscin, and pseudoprotogracillin from DNR. Then, the extraction conditions for the four steroidal saponins, including the water content of NADESs, extraction time, and liquid-solid ratio were systematically optimized using response surface methodology (RSM), and the microstructure of the raw and extracted DNR powder were observed by field emission scanning electron microscope. Moreover, the recovery of the four steroidal saponins from tailored NADESs was tentatively carried out by D101 macroporous resins.

\section{Results and Discussion}

\subsection{HPLC Conditions and Method Validation}

In order to achieve a rapid and efficient analysis of the four bioactive steroidal saponins (protodioscin, protogracillin, pseudoprotodioscin, and pseudoprotogracillin) in DNR, several HPLC conditions, including mobile phases (water-methanol, water-acetonitrile, formic acid water-methanol, and formic acid water-acetonitrile), flow rates $(0.9,1.0$, and $1.1 \mathrm{~mL} / \mathrm{min})$, and column temperatures $\left(20,30\right.$, and $\left.40^{\circ} \mathrm{C}\right)$ were compared and optimized. As a result, the water-acetonitrile system at $30^{\circ} \mathrm{C}$ with a flow rate of $1.0 \mathrm{~mL} / \mathrm{min}$ was selected for the suitable analysis duration, greater separation ability, and better peak shapes of the four analytes. The chromatographic peaks of: protodioscin (1), protogracillin (2), pseudoprotodioscin (3), and pseudoprotogracillin (4) were confirmed by comparing their retention times with the reference standards. The typical HPLC chromatograms of DNR sample and the four steroidal saponins reference standards are shown in Figure 1. 

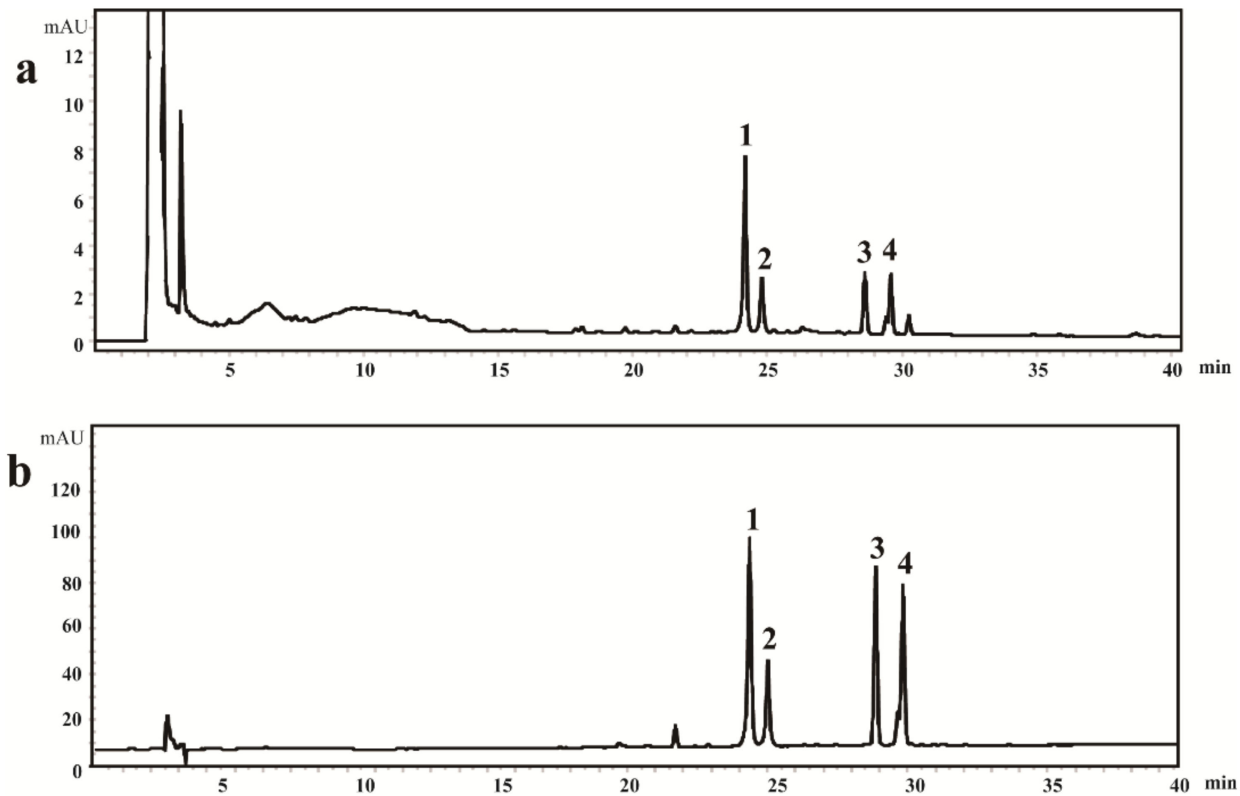

Figure 1. The typical HPLC chromatograms of: (a) Dioscoreae Nipponicae Rhizoma sample and (b) four steroidal saponins reference standards. (1. protodioscin, $24.15 \mathrm{~min}$; 2. protogracillin, $24.77 \mathrm{~min}$; 3. pseudoprotodioscin, $28.60 \mathrm{~min}$; and 4. Pseudoprotogracillin, $29.56 \mathrm{~min}$ ).

Method validation of quantitative analysis of the four bioactive steroidal saponins in DNR was performed. The linearity, limit of detections (LODs), limit of quantifications (LOQs), precision, repeatability, stability, and accuracy were validated. The calibration curves of the four steroidal saponins were performed with six different concentrations of standard solutions in triplicate. All calibration curves were of good linearity with high correlation coefficient $\left(r^{2}>0.9992\right)$ over the tested range. The LODs and LOQs of the four analytes were defined by the concentration that generated peaks with signal-to-noise values of 3 and 10 using standard solutions. The precision of the method was determined by the intra- and inter-day variations. For intra-day tests, the same sample was analyzed six times within the same day, while for inter-day tests, the sample was examined in duplicates for three consecutive days. The relative standard deviations (RSDs) of intra- and inter-day precisions were less than $1.4 \%$ and $2.7 \%$, respectively. For the repeatability test, six replicates of the same sample were prepared and analyzed, and for the stability test, the same sample was stored at room temperature and analyzed by replicate injection analysis at $0,2,4,8,12$, and $24 \mathrm{~h}$. The repeatability presented as RSDs were less than $2.1 \%$, and the stability were less than $3.0 \%$. The recovery test was used to evaluate the accuracy of the method. A known amount of the four steroidal saponins reference standard solutions were added into the same samples in sextuplicate, and then the samples were extracted and analyzed with the same procedures as described in Section 3.3. The recovery of each analyte was calculated by the equation: Recovery $(\%)=($ Detected amount - Original amount)/Spiked amount $\times 100 \%$. The overall recoveries of the four analytes were in the range of $98.8-107.5 \%$ with RSDs less than $2.3 \%$. All of the above data are shown in Table 1, which indicates the established method is precise, accurate, and sensitive for the quantification of the four steroidal saponins in DNR samples. 
Table 1. Regression equation, limit of detections (LODs), limit of quantifications (LOQs), precision, repeatability, stability, and recovery of four bioactive steroidal saponins.

\begin{tabular}{|c|c|c|c|c|c|c|c|c|c|c|c|}
\hline \multirow[b]{2}{*}{ No. } & \multirow[b]{2}{*}{ Analyte } & \multirow[b]{2}{*}{ Regression Equation } & \multirow[b]{2}{*}{$r^{2}$} & \multirow{2}{*}{$\begin{array}{c}\text { Linear } \\
\text { Range } \\
(\mathrm{mg} / \mathrm{mL})\end{array}$} & \multirow{2}{*}{$\begin{array}{l}\text { LODs } \\
(\mu \mathrm{g} / \mathrm{mL})\end{array}$} & \multirow{2}{*}{$\begin{array}{l}\text { LOQs } \\
(\mu \mathrm{g} / \mathrm{mL})\end{array}$} & \multicolumn{2}{|c|}{ Precision } & \multirow{2}{*}{$\begin{array}{l}\text { Repeatability } \\
\text { RSD (\%) }\end{array}$} & \multirow{2}{*}{$\begin{array}{l}\text { Stability } \\
\text { RSD (\%) }\end{array}$} & \multirow{2}{*}{$\begin{array}{c}\text { Recovery } \\
(\text { mean } \pm S D, \%)\end{array}$} \\
\hline & & & & & & & $\begin{array}{c}\text { Intra-Day } \\
\text { RSD (\%) }\end{array}$ & $\begin{array}{c}\text { Inter-Day } \\
\text { RSD (\%) }\end{array}$ & & & \\
\hline 1 & protodioscin & $Y=1657.1 X+41.135$ & 0.9993 & $0.050-0.84$ & 0.054 & 0.18 & 1.1 & 2.2 & 1.6 & 1.2 & $104.7 \pm 1.6$ \\
\hline 2 & protogracillin & $\mathrm{Y}=1067.8 \mathrm{X}+20.476$ & 0.9997 & $0.030-0.49$ & 0.076 & 0.26 & 1.4 & 2.7 & 1.1 & 3.0 & $104.8 \pm 2.4$ \\
\hline 3 & pseudoprotodioscin & $\mathrm{Y}=3978.2 \mathrm{X}-100.22$ & 0.9992 & $0.040-0.64$ & 0.048 & 0.16 & 1.2 & 2.1 & 2.1 & 2.6 & $107.5 \pm 1.9$ \\
\hline 4 & pseudoprotogracillin & $Y=3549.6 X-32.319$ & 0.9997 & $0.030-0.44$ & 0.037 & 0.12 & 1.3 & 2.4 & 1.7 & 1.7 & $98.8 \pm 1.9$ \\
\hline
\end{tabular}




\subsection{Preparation of NADESs}

In general, the extraction efficiency for bioactive compounds from plant sources could potentially be influenced by both the extraction conditions and the physicochemical properties of the extraction solvents. Similarly, NADESs are composed of a mixture consisting of HBAs and HBDs of natural origins, and the chemical compositions of NADESs determines their physicochemical properties and consequently greatly influences extraction efficiency of bioactive compounds from natural products. In this work, with the aim to select the most appropriate NADES for the extraction of bioactive steroidal saponins, three groups of HBDs (alcohols, carboxylic acid, and amides) were used in combinations with three types of HBAs (choline chloride, betaine, and L-PROLINE) to prepare NADESs. A total of thirty combinations of NADESs were initially prepared, and twenty-one of them were eventually found to be stable as a clear, viscous liquid without precipitation over the course of time (Table 2).

During the preparation of different types of NADESs, we found that the chemical structures of HBAs play essential roles in the formation and stability of NADESs. NADESs could be successfully formed by heating $\mathrm{ChCl}$ or Bet with lactic acid or malonic acid, in contrast, Pro was proved incapable of producing a stable liquid when heated with lactic acid or malonic acid. Amides-based NADESs could be prepared when the HBAs was $\mathrm{ChCl}$, but when the HBAs were Bet and Pro, amides-based NADESs could not successfully be formed. When HBDs were in a liquid form, such as glycerol and ethylene glycol, it was easier to synthesize a clear and less viscous NADESs, and the carboxylic acid-based NADESs were also easier to synthesize. In addition, the different ratios of HBAs and HBDs might affect the synthesis and stability of NADESs. For example, when $\mathrm{ChCl}$ were heated with malonic acid in the mole ratios of 2:1, 1.5:1, 1:1, 1:1.5, and 1:2, a clear liquid can be only prepared with $1.5: 1,1: 1$, or 1:1.5 mole ratio.

Table 2. List of natural deep eutectic solvents (NADESs) synthesized in this study.

\begin{tabular}{ccccc}
\hline No. & Abbreviation & $\begin{array}{c}\text { Hydrogen Bond } \\
\text { Acceptors (HBAs) }\end{array}$ & $\begin{array}{c}\text { Hydrogen Bond Donors } \\
\text { (HBDs) }\end{array}$ & Mole Ratios \\
\hline 1 & ChCl-Eg & Choline chloride & Ethylene glycol & $1: 2$ \\
2 & ChCl-Gly & Choline chloride & Glycerol & $1: 2$ \\
3 & ChCl-Maa & Choline chloride & DL-Malic acid & $1: 1$ \\
4 & ChCl-Lac & Choline chloride & Lactic acid & $1: 1$ \\
5 & ChCl-Mal & Choline chloride & Malonic acid & $1: 1$ \\
6 & ChCl-Mu & Choline chloride & 1-Methylurea & $1: 1$ \\
7 & ChCl-Am & Choline chloride & Acetamide & $1: 1$ \\
8 & ChCl-Ur & Choline chloride & Urea & $1: 2$ \\
9 & Bet-Xyl & Betaine & Xylitol & $1: 1$ \\
10 & Bet-Gly & Betaine & Glycerol & $1: 2$ \\
11 & Bet-Sor & Betaine & D-Sorbitol & $1: 1$ \\
12 & Bet-Maa & Betaine & DL-Malic acid & $1: 1$ \\
13 & Bet-Lac & Betaine & Lactic acid & $1: 1$ \\
14 & Bet-Mal & Betaine & Malonic acid & $1: 1$ \\
15 & Bet-Ca & Betaine & Citric acid & $2: 1$ \\
16 & Pro-Gly & L-proline & Glycerol & $5: 2$ \\
17 & Pro-Sor & L-proline & D-Sorbitol & $1: 2$ \\
18 & Pro-Xyl & L-proline & Xylitol & $1: 1$ \\
19 & Pro-Ca & L-proline & Citric acid & $1: 1$ \\
20 & Pro-Ox & L-proline & Oxalic acid & $1: 1$ \\
21 & Pro-Maa & L-proline & DL-Malic acid & $1: 1$ \\
\hline
\end{tabular}

\subsection{Screening of NADESs for Extraction of Bioactive Steroidal Saponins}

Compared with conventional extraction solvents, the high viscosity of NADESs restricted their application in extraction. High viscosity may impair the efficiency of extraction as slow mass transfer occurs, and the low viscosity of the NADESs led to high diffusivity and hence improved the extraction performance [22]. In the initial screening 
experiments, extraction conditions were adjusted to reduce the viscosity by adding a certain amount of water. It is reported that a large excess of water could break the hydrogen bonds between the NADESs components and lose the eutectic property of the solvents produced [23]. Therefore, the NADESs were diluted to $70 \%(v / v)$ with water to decrease viscosities for enhancing the extraction efficiency.

Extraction yields of the four steroidal saponins (protodioscin, protogracillin, pseudoprotodioscin, and pseudoprotogracillin) and the total four steroidal saponins with different types of NADESs are shown in Figure 2. The results of initial screening experiments indicated that the extraction efficiency for the four bioactive steroidal saponins was influenced by the types of NADESs solvents, and different types of DESs resulted in different extraction yields. In general, extraction yields of the four steroidal saponins followed the order protodioscin $>$ protogracillin $>$ pseudoprotodioscin $>$ pseudoprotogracillin. It was obvious that protodioscin showed higher extraction efficiency in ChCl-Lac, followed by $\mathrm{ChCl}-\mathrm{Maa}, \mathrm{ChCl}-\mathrm{Mu}$, and Bet-Lac. Similarly, protogracillin exhibited higher extraction efficiency in ChCl-Lac, ChCl-Maa, ChCl-Mu, and Bet-Lac. For pseudoprotodioscin and pseudoprotogracillin, NADESs ChCl-Mal, ChCl-Maa, and Pro-Xyl led to higher extraction yields. The total extraction yields of the four steroidal saponins were also calculated, and $\mathrm{ChCl}-\mathrm{Mal}$ and $\mathrm{ChCl}-\mathrm{Lac}$ showed relative higher extraction efficiency for the four bioactive steroidal saponins compared with other NADESs.
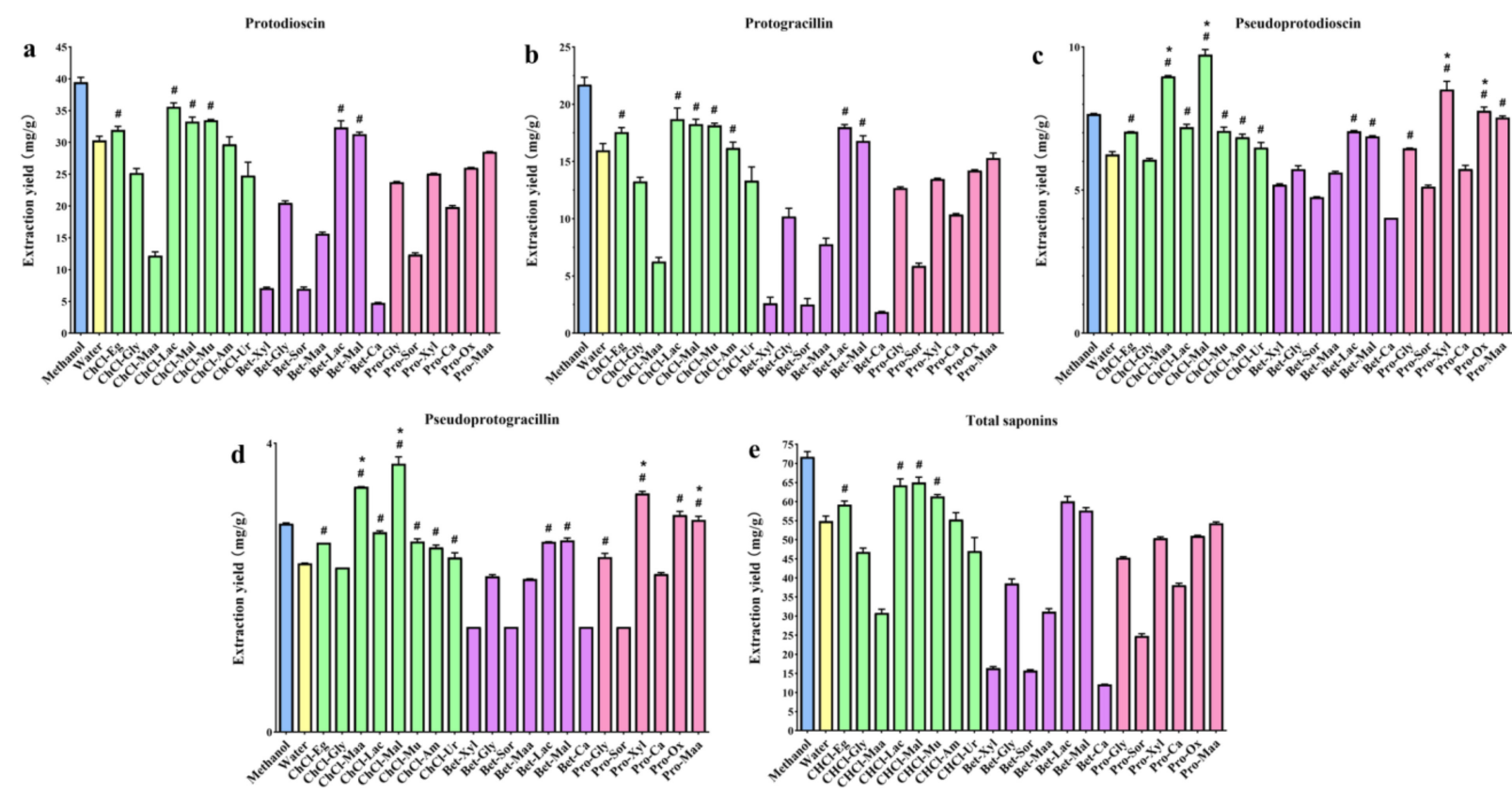

Figure 2. Extraction yields of different solvents for (a) protodioscin, (b) protogracillin, (c) pseudoprotodioscin, (d) pseudoprotogracillin, and (e) four total steroidal saponins. Error bars indicate the standard deviations $(n=3)$. Extraction yields of NADESs compared with water are indicated with $\# p<0.05$, mean value $>$ water and ${ }^{*} p<0.05$, mean value $>$ methanol.

To get insight into the NADESs' excellence in the extraction of bioactive steroidal saponins from DNR, the extraction yields of different NADESs and conventional efficient solvents (methanol and water) were compared. For protodioscin and protogracillin, almost all the NADESs exhibited lower extraction efficiency compared with methanol, and the $\mathrm{ChCl}-\mathrm{Eg}$, ChCl-Lac, ChCl-Mal, ChCl-Mu, Bet-Lac, and Bet-Mal exhibited higher extraction efficiency compared with water. For pseudoprotodioscin and pseudoprotogracillin, the $\mathrm{ChCl}-\mathrm{Maa}, \mathrm{ChCl}-\mathrm{Mal}$, Pro-Xyl, and Pro-Ox exhibited higher extraction efficiency compared with methanol and water. For the total four steroidal saponins, the extraction efficiency of ChCl-Eg, ChCl-Lac, ChCl-Mal, ChCl-Mu, ChCl-Am, Bet-Lac, and Bet-Mal was higher than water with the concentration $59.20 \pm 0.02,64.27 \pm 0.03,64.99 \pm 0.02,61.36 \pm 0.01$, $55.31 \pm 0.03,60.05 \pm 0.02$, and $57.64 \pm 0.01 \mathrm{mg} / \mathrm{g}$, respectively. Compared to methanol, 
most NADESs showed lower extraction yields of the total four steroidal saponins, but $\mathrm{ChCl}-\mathrm{Lac}(64.27 \pm 0.03 \mathrm{mg} / \mathrm{g})$ and ChCl-Mal $(64.99 \pm 0.02 \mathrm{mg} / \mathrm{g})$ produced approximate extraction efficiency. Thus, ChCl-Mal was selected as the best NADES for extraction of steroidal saponins from DNR and applied in further tests based on the initial screening results.

\subsection{Optimization of the Extraction Conditions for Bioactive Steroidal Saponins}

\subsubsection{Optimization of Molar Ratios of ChCl-Mal}

The molar ratio of HBAs and HBDs has been regarded as an important factor that influences the surface tension and polarity of NADESs [24], which could affect the extraction efficiency of target components from natural biomass. As described above, $\mathrm{ChCl}-\mathrm{Mal}$ produced the highest extraction efficiency of steroidal saponins from DNR. The molar ratios of ChCl-Mal were further investigated. The eutectic mixtures of ChCl-Mal were prepared at mole ratios of 2:1, 1.5:1, 1:1, 1:1.5, and 1:2, and homogeneous and transparent liquid could be synthesized with 1.5:1, 1:1, or 1:1.5 mole ratios. Thus, the ChCl-Mal with the mole ratios 1.5:1, 1:1, and 1:1.5 were used to extract bioactive steroidal saponins from DNR. As shown in Figure 3, the results indicated that the different molar ratios of $\mathrm{ChCl}-\mathrm{Mal}$ have impact on the extraction yields of protodioscin and protogracillin, but have no impact on pseudoprotodioscin and pseudoprotogracillin. The optimal molar ratio of $\mathrm{ChCl}-\mathrm{Mal}$ was 1:1 and the extraction yields of protodioscin, protogracillin, pseudoprotodioscin, and pseudoprotogracillin were $29.30 \pm 0.67,15.86 \pm 0.37,9.71 \pm 0.23$, and $3.66 \pm 0.07 \mathrm{mg} / \mathrm{g}$, respectively.

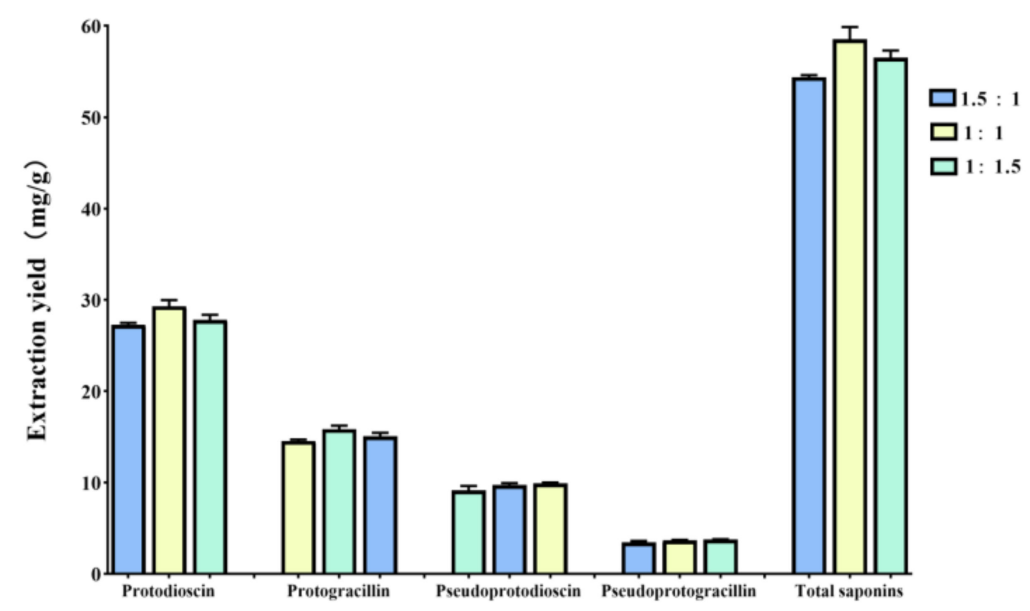

Figure 3. Extraction yields of different molar ratios (1.5:1, 1:1, and 1:1.5) of ChCl-Mal for protodioscin, protogracillin, pseudoprotodioscin, pseudoprotogracillin, and four total steroidal saponins. Error bars indicate the standard deviation $(n=3)$.

\subsubsection{Optimization of the Extraction Conditions by Response Surface Methodology}

RSM was an effective method to design and analyze tests with independent variables upon different levels to optimize the results, and had been widely used in optimization of extraction processes $[25,26]$. The above extraction investigations showcased that $\mathrm{ChCl}-\mathrm{Mal}$ with the mole ratio 1:1 was the best NADES for extraction of bioactive steroidal saponins from DNR. In order to obtain the optimal extraction efficiency for steroidal saponins, several extraction conditions that could affect the extraction efficiencies were optimized by RSM. Similar to previous researches, three independent variables, including water content in NADESs, extraction time, and liquid-solid ratios were further optimized. After determining the range of extraction factors on the basis of preliminary single-factor tests, the water content in NADESs (A, 20-40\%), extraction time (B, 10-40 min), and liquidsolid ratios $(\mathrm{C}, 10-50 \mathrm{~mL} / \mathrm{g})$ at three level $(-1,0,1)$ in the selected NADES (ChCl-Mal) were evaluated using Box-Behnken design (BBD). The extraction yields of four bioactive 
steroidal saponins were set as the response of the design experiments. The experimental orders, levels of variables, and response values are summarized in Table 3.

Table 3. The experimental orders, levels of variables, and response values in Box-Behnken design.

\begin{tabular}{ccccc}
\hline Run. & $\begin{array}{c}\text { Water Content in NADESs } \\
(\mathbf{A}, \mathbf{\%})\end{array}$ & $\begin{array}{c}\text { Extraction Time } \\
(\mathbf{B}, \mathbf{m i n})\end{array}$ & $\begin{array}{c}\text { Liquid-Solid Ratio } \\
(\mathbf{C}, \mathbf{~ m L} / \mathbf{g})\end{array}$ & $\begin{array}{c}\text { Total Extraction Yields of Four } \\
\text { Steroidal Saponins }(\mathbf{m g} / \mathbf{g})\end{array}$ \\
\hline 1 & $40(1)$ & $25(0)$ & $50(1)$ & 61.69 \\
2 & $30(0)$ & $40(1)$ & $10(-1)$ & 58.15 \\
3 & $40(1)$ & $25(0)$ & $10(-1)$ & 56.01 \\
4 & $40(1)$ & $40(1)$ & $30(0)$ & 64.55 \\
5 & $20(-1)$ & $10(-1)$ & $30(0)$ & 54.62 \\
6 & $30(0)$ & $25(0)$ & $30(0)$ & 63.25 \\
7 & $30(0)$ & $25(0)$ & $30(0)$ & 66.21 \\
8 & $30(0)$ & $10(-1)$ & $50(1)$ & 53.81 \\
9 & $20(-1)$ & $40(1)$ & $30(0)$ & 66.79 \\
10 & $20(-1)$ & $25(0)$ & $10(-1)$ & 45.14 \\
11 & $20(-1)$ & $25(0)$ & $50(1)$ & 56.36 \\
12 & $30(0)$ & $25(0)$ & $30(0)$ & 63.13 \\
13 & $30(0)$ & $25(0)$ & $30(0)$ & 58.59 \\
14 & $40(1)$ & $10(-1)$ & $30(0)$ & 66.13 \\
15 & $30(0)$ & $40(1)$ & $50(1)$ & 56.28 \\
17 & $30(0)$ & $10(-1)$ & $30(0)$ & 65.65 \\
\end{tabular}

The variables and response were processed to build a mathematical regression model for steroidal saponins. The model was expressed as second order polynomial quadratic equation for the extraction yields $(\mathrm{Y})$ and coded variables $(\mathrm{A}, \mathrm{B}$, and $\mathrm{C})$ as follows:

$\mathrm{Y}=63.27+3.18 \mathrm{~A}+3.07 \mathrm{~B}+2.77 \mathrm{C}-3.44 \mathrm{AB}-1.38 \mathrm{AC}-2.26 \mathrm{BC}+0.41 \mathrm{~A}^{2}-0.66 \mathrm{~B}^{2}-0.89 \mathrm{C}^{2}$

The quality of regression model for steroidal saponins was evaluated in terms of the square of correlation coefficient $\left(R^{2}\right)$ and the Lack of Fit by the analysis of variance (ANOVA) at the 95\% confidence level. As shown in Table 4, the $\mathrm{R}^{2}$ value was 0.9153 , indicating that the experimental data were in relatively good agreement with predicted extraction yields. The Lack of Fit value was insignificant for the response with $p$-value of $0.4976(p>0.05)$, which indicated that the regression model fitted the models well and could suitably explain the data.

Table 4. The analysis of variance (ANOVA) results of the quadratic multiple regression model for steroidal saponins.

\begin{tabular}{cccccc}
\hline Variables & Sum of Squares & df & Mean Square & F-Value & $p$-Value \\
\hline Model & 631.18 & 9 & 70.13 & 8.40 & 0.0052 \\
A-Water content & 81.09 & 1 & 81.09 & 9.02 & 0.0169 \\
B-Extraction time & 75.31 & 1 & 75.31 & 7.37 & 0.0198 \\
C-Liquid-solid ratio & 67.51 & 1 & 61.51 & 5.66 & 0.0300 \\
AB & 47.25 & 1 & 47.25 & 7.92 & 0.0489 \\
AC & 7.65 & 1 & 7.65 & 0.087 & 0.3703 \\
BC & 20.42 & 1 & 20.42 & 0.22 & 0.7767 \\
A $^{2}$ & 0.73 & 1 & 1.85 & 39.84 & 0.6520 \\
B $^{2}$ & 1.85 & 1 & 332.50 & 0.95 & 0.0004 \\
C $^{2}$ & 332.50 & 1 & 8.35 & & 0.4976 \\
Lack of Fit & 58.42 & 7 & 8.09 & & \\
R $^{2}$ & 24.26 & 3 & & &
\end{tabular}


Statistical analysis and 3D response plots (Figure 4) obviously illustrated the significant variables affecting extraction yields of steroidal saponins and the interaction effects between the variables. It was clear that all the three variables, water content in NADESs, extraction time, and liquid-solid ratios showed significant effects on the extraction efficiency of steroidal saponins $(p<0.05)$. Based on the regression model, the optimum condition of $\mathrm{ChCl}-\mathrm{Mal}$ for extraction of four bioactive steroidal saponins from DNR was as following: water content in NADESs, 39.69\%; extraction time, 19.98 min; liquid-solid ratios, $32.28 \mathrm{~mL} / \mathrm{g}$. For the convenience of practical operation, the optimum condition was set at water content in NADESs, $40 \%$; extraction time, 20 min; liquid-solid ratios, $32 \mathrm{~mL} / \mathrm{g}$. Triplicate experiments were carried out under the optimal extraction conditions and mean values of experimental results were compared with the predicted values. Under the optimum conditions, the actual extraction yield of the total four steroidal saponins was $66.82 \mathrm{mg} / \mathrm{g}$, which was closed to the predicted extraction yield of $66.89 \mathrm{mg} / \mathrm{g}$. This results indicated that the model was credible for optimization of extraction parameters of steroidal saponins from DNR.
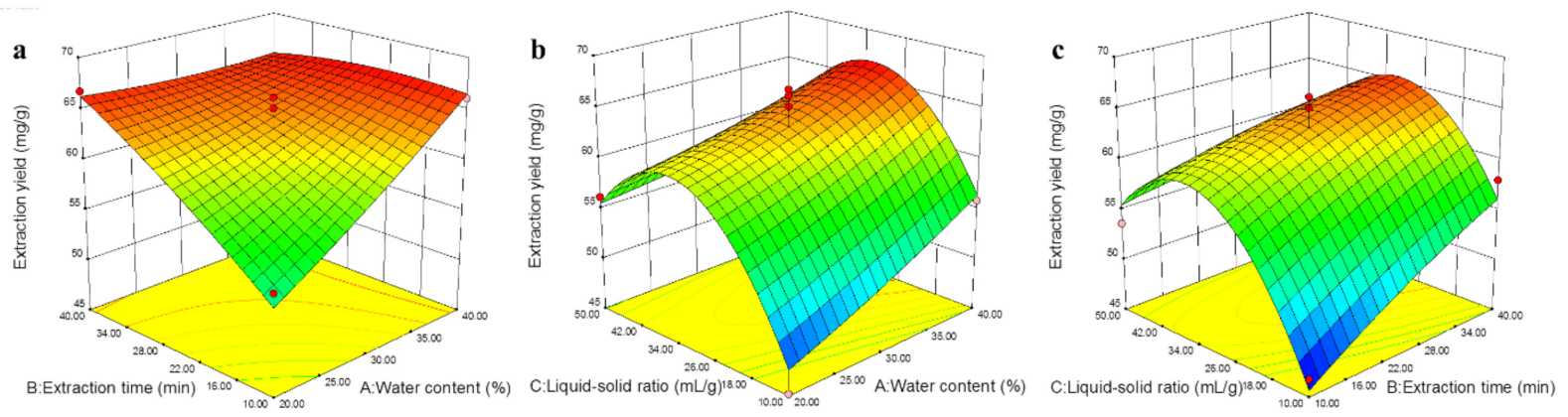

Figure 4. Response surface plots of the model for extraction of steroidal saponins from Dioscoreae Nipponicae Rhizoma (DNR). (a) water content (\%) and extraction time (min); (b) water content (\%) and liquid-solid ratio (mL/g); (c) liquid-solid ratio $(\mathrm{mL} / \mathrm{g})$ and extraction time $(\mathrm{min})$.

\subsection{Microstructure of Plant Material}

Several previous studies have been reported that the NADESs could replace the organic solvents as extraction media for extraction of bioactive compounds from natural products due to their great extraction performance, which agrees with the results of this study $[27,28]$. However, the explanation of the superior extraction performance of some NADESs are still unclear. It is well known that the ultrasonic extraction could utilize the ultrasound irradiation energy to produce cavitation phenomenon, along with the formation of bubbles that collapsed in the multiple directions of the plant surface. This external force could break the plant cell wall and reduce the particle size, facilitating swelling, and extraction solvent permeation into the cells to release the bioactive compounds from the vacuole [29].

In the present study, the microstructures of the DNR powder before and after ultrasonic extraction in water, methanol, and the selected NADES (ChCl-Mal) were observed by field emission scanning electron microscope. As shown in Figure 5, the DNR powder before ultrasonic extraction displayed a rough and coarse surface with many flakes (Figure 5a), whereas the surface of powder after ultrasonic extraction in water, methanol, and $\mathrm{ChCl}$ Mal displayed some breakages along the edges without any flakes (Figure $5 b-d$ ). The results illustrated that both the conventional solvents (water and methanol) and NADES (ChCl-Mal) could destroy the cells and cell walls as well as dissolved those weak flakes during ultrasonic extraction [30]. 


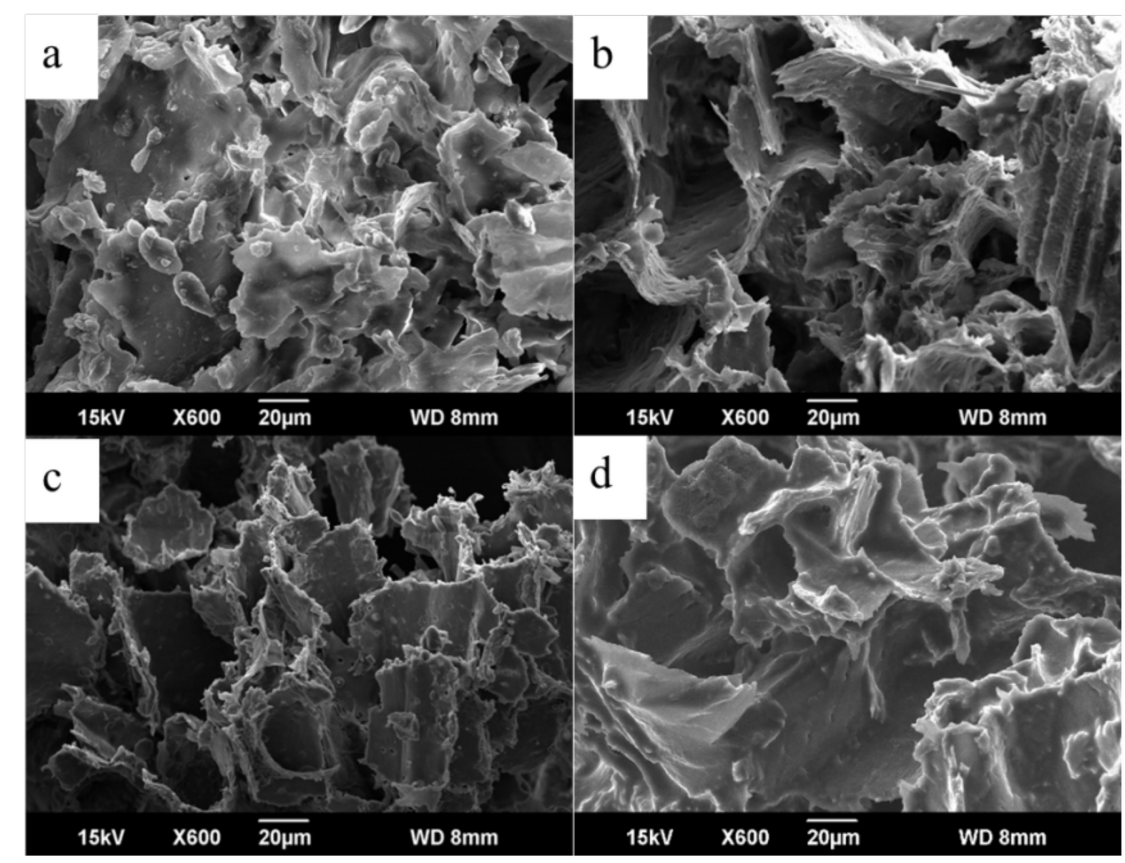

Figure 5. The field emission scanning electron microscope images of DNR powder: (a) before ultrasonic extraction and after ultrasonic extraction in (b) water, (c) methanol, and (d) the selected NADES (ChCl-Mal).

\subsection{Recovery of Bioactive Steroidal Saponins from NADESs}

The recovery of extracted bioactive compounds from NADESs is a challenge due to the high water miscibility and non-volatilization property of NADESs. The D101 macroporous resin is a common, accessible, and inexpensive packing material for column chromatography. In this study, the recovery of the four bioactive steroidal saponins from ChCl-Mal was tentatively applied by using D101 macroporous resin column chromatography according to our previous research [31]. As described in Section 3.8, the recoveries of the four bioactive steroidal saponins, protodioscin, protogracillin, pseudoprotodioscin, and pseudoprotogracillin were $79.90 \%, 68.12 \%, 67.27 \%$, and $74.8 \%$, respectively. The results indicated that D101 macroporous resin could effectively adsorb steroidal saponins while the polar ingredients of NADESs could be elected with water, and most steroidal saponins could be obtained with ethanol after eluting the polar compounds with water. In summary, the recovery of the four steroidal saponins in NADESs extraction solution could be conveniently, readily, and efficiently achieved using D101 microporous resin.

\section{Materials and Methods}

\subsection{Materials and Reagents}

DNR samples were purchased from a local Traditional Chinese Medicine market (Anguo, Hebei, China). The samples were identified by Associate Professor Long Guo, and the voucher specimens have been deposited in Hebei University of Chinese Medicine, Shijiazhuang, China. The DNR samples were dried in the shade and powered by a disintegrator, then sieved through 60 mesh and stored in the desiccator before use.

Choline chloride $(\mathrm{ChCl})$, betaine (Bet), L-proline (Pro), ethylene glycol (Eg), glycerol (Gly), xylitol (Xyl), D-sorbitol (Sor), lactic acid (Lac), malonic acid (Mal), DL-malic acid (Maa), Oxalic acid (Ox), Citrate acid (Ca), 1-Methylurea (Mu), Acetamide (Am), and Urea (Ur) were purchased from Aladdin Biochemical Technology (Shanghai, China). The reference compounds of protodioscin, protogracillin, pseudoprotodioscin, and pseudoprotogracillin (purities $>95 \%$ ) were obtained from Chengdu Biopurify Phytochemicals Company (Chengdu, China). The chemical structures of the four steroidal saponins are shown in Figure 6. Acetonitrile and methanol (HPLC grade) were purchased from Fisher 
Scientific (Geel, Belgium). Deionized water was prepared by a Synergy water purification system (Millipore, Billerica, USA). Other reagents and chemicals were of analytical grade.

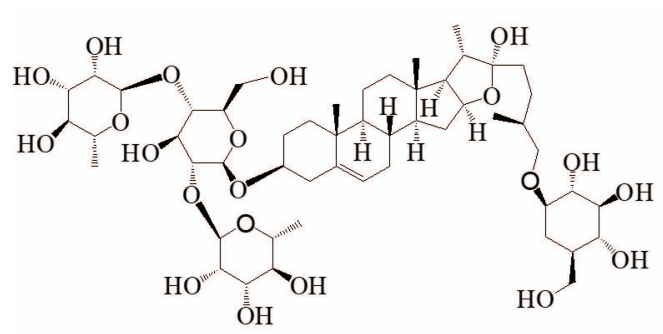

Protodioscin

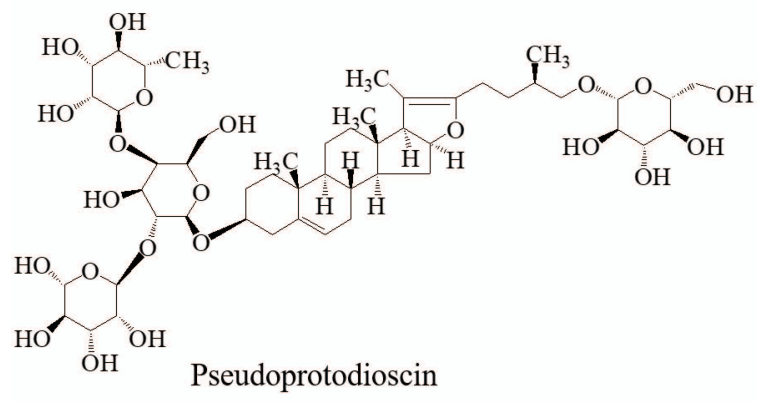

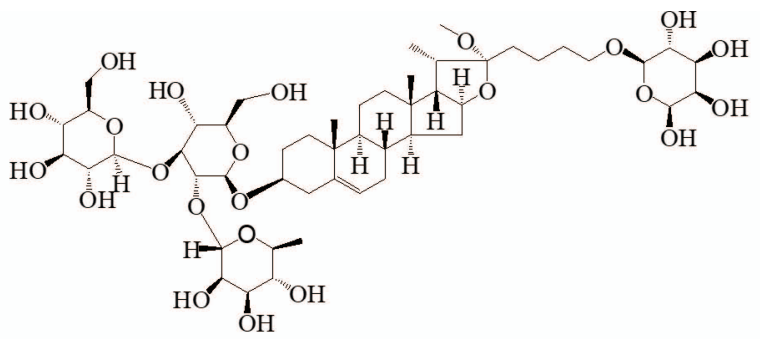

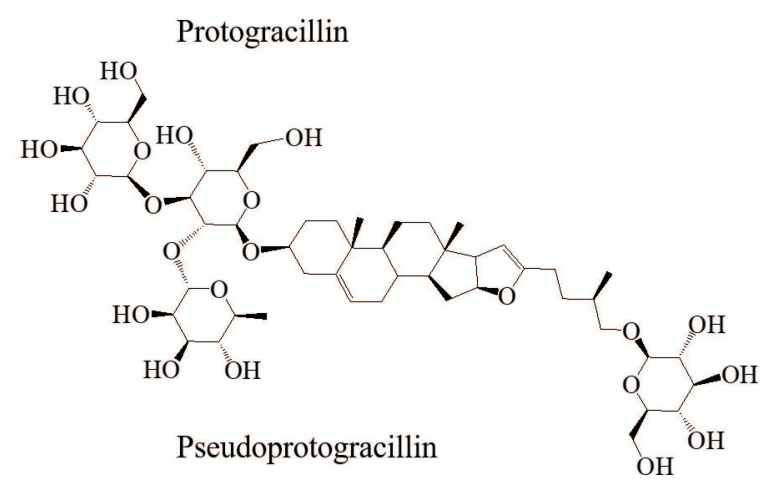

Figure 6. Chemical structures of protodioscin, protogracillin, pseudoprotodioscin, and pseudoprotogracillin.

\subsection{Preparation of NADESs}

NADESs can be synthesized by a heating method according to the previous study [31] Briefly, DESs were synthesized by simply mixing the hydrogen bond acceptors (HBAs) and hydrogen bond donors (HBDs) at a proper molar ratio with magnetic agitation at $80.0^{\circ} \mathrm{C}$ until a homogeneous and transparent liquid formed.

\subsection{Extraction Procedures}

For initial screening of NADESs for extraction of the four bioactive steroidal saponins from DNR, an accurately weighed DNR powder $(50 \mathrm{mg})$ was extracted with $1 \mathrm{~mL}$ of prepared NADESs containing $30 \%$ water in a $2-\mathrm{mL}$ centrifuge tube by ultrasonic ( $300 \mathrm{~W}$, $40 \mathrm{kHz}$ ) for $20 \mathrm{~min}$ at room temperature. After extraction, the mixed suspension was centrifuged at 13,000 $\mathrm{rpm} / \mathrm{min}$ for $10 \mathrm{~min}$. Then, the supernatant was diluted eight times using 50\% methanol and filtered through membrane filters $(0.22 \mu \mathrm{m})$ prior to HPLC analysis. Each extraction was performed in triplicate.

\subsection{Preparation of Standard Solutions}

The reference compounds of protodioscin, protogracillin, pseudoprotodioscin, and pseudoprotogracillin were accurately weighed and dissolved in DES (ChCl-Mal), and then diluted eight times with $50 \%$ methanol to the concentrations of $840,490,640$, and $320 \mu \mathrm{g} / \mathrm{mL}$, respectively. The standard solutions containing four reference standards were prepared by appropriate dilution to a series of proper concentrations. The standard solutions were stored at $4{ }^{\circ} \mathrm{C}$ for further HPLC analysis.

\subsection{Determination of Bioactive Steroidal Saponins by HPLC}

The concentrations of four steroidal saponins extracted by NADESs were determined using an Agilent 1260 HPLC system (Agilent, San Jose, CA, USA) equipped with a quaternary pump, an autosampler, a thermostatic column compartment and a diode array detector. Separation was performed on an Agilent ZORBAX Eclipse Plus C18 column $(4.6 \mathrm{~mm} \times 250 \mathrm{~mm}, 5 \mu \mathrm{m})$. The mobile phase consisted of water (A) and acetonitrile (B), and the gradient conditions were as follows: $0-5 \mathrm{~min}, 15 \% \mathrm{~B} ; 5-30 \mathrm{~min}, 15-40 \% \mathrm{~B}$; and 
30-50 $\mathrm{min}, 40-50 \% \mathrm{~B}$. The column temperature was set at $30{ }^{\circ} \mathrm{C}$, and the flow rate was set at $1.0 \mathrm{~mL} / \mathrm{min}$. The detection wavelength was $203 \mathrm{~nm}$, and the injection volume was $20 \mu \mathrm{L}$.

\subsection{Optimization of the Extraction Conditions for Bioactive Steroidal Saponins}

The extraction parameters of the bioactive steroidal saponins by the selected NADES were optimized using RSM, which is a statistical technique for studying the interactions between factors in an appropriate variable range. On the basis of preliminary singlefactor experiments, the water content (\%) in NADESs (A), extraction time (B), and liquidsolid ratios $(\mathrm{C})$ were selected as three independent variables to optimize the extraction parameters by Box-Behnken design (BBD). The total extraction yields of the four bioactive steroidal saponins were taken as the response of the design experiments. Regression analysis was performed according to the experimental data, and additional confirmation experiments were conducted to confirm the validity of the statistical experimental strategies. The Design-Expert Ver. 8.0.6 software (Stat-Ease Inc., Minneapolis, MN, USA) was used for the generation and evaluation of the experimental design.

\subsection{Microstructure of Plant Material}

A JEOL (JEOL Ltd., Tokyo, Japan) JSM-6390A field emission scanning electron microscope in the secondary electron mode with $15-\mathrm{kV}$ accelerating voltage was used for observing the microstructure of the raw and extracted DNR powder. Before imaging, the samples were coated with a Pt layer of approximately 10-nm thickness.

\subsection{Recovery of Bioactive Steroidal Saponins from NADESs}

Recovery of the four steroidal saponins from the NADESs was conducted based on microporous resin column chromatography. A glass column filled with $10 \mathrm{~g}$ D101 macroporous resin was used and the bed volume (BV) was $20 \mathrm{~mL}$. The NADESs solution $(1 \mathrm{~mL})$ was loaded at the flow rate of $3 \mathrm{BV} / \mathrm{h}$. After the target compounds have been adsorbed in the resin, the column was washed with deionized water $(100 \mathrm{~mL})$ first, and then eluted with $100 \mathrm{~mL}$ ethanol. Then, the ethanolic fraction was concentrated under vacuum, and analyzed by HPLC as described in Section 3.4. The recoveries of four steroidal saponins were calculated and the above experiments were carried out by triplicate.

\section{Conclusions}

In the present study, a green and efficient extraction method using NADESs as the extraction solvent was established for extraction of bioactive steroidal saponins from DNR. A total of twenty-one types of NADESs were successfully synthesized and used to extract four bioactive steroidal saponins, including protodioscin, protogracillin, pseudoprotodioscin, and pseudoprotogracillin from DNR. The results indicated that the tailor-made NADESs were efficient solvents for the extraction of steroidal saponins, and the ChCl-Mal with a molar ratio of 1:1 proved to be the most efficient NADES. Then, the extraction parameters of ChCl-Mal for extraction of the four bioactive steroidal saponins were optimized using RSM and the optimal extraction condition was water content in NADESs, $40 \%$; extraction time, $20 \mathrm{~min}$; and liquid-solid ratios, $32 \mathrm{~mL} / \mathrm{g}$. Moreover, the microstructure of the DNR powder before and after ultrasonic extraction were observed by field emission scanning electron microscope. The recovery of the extracted steroidal saponins from NADESs was using D101 macroporous resin and the recovery yields of protodioscin, protogracillin, pseudoprotodioscin, and pseudoprotogracillin were $79.90 \%, 68.12 \%, 67.27 \%$, and $74.8 \%$, respectively. The present research suggests that NADESs are efficient solvents which could be used as green media for extraction of bioactive steroidal saponins from DNR, and the data acquired in this study might contribute to further NADESs application in extraction of bioactive compounds from natural products.

Author Contributions: Conceptualization, L.G. and D.Z.; methodology, G.-Y.Y. and J.-N.S.; validation, G.-Y.Y., J.-N.S., and Y.-Q.C.; resources, Y.-G.Z.; data curation, L.W.; writing-original draft 
preparation, G.-Y.Y.; writing-review and editing, L.G. and L.W. All authors have read and agreed to the published version of the manuscript.

Funding: This research was funded by National Natural Science Foundation of China (81803697), Natural Science Foundation of Hebei Province (H2018423032), Research Foundation of Hebei Province Education Department (BJ2019006), Research Foundation of Hebei Provincial Administration of Traditional Chinese Medicine (2018108) and Innovation Team of Hebei Province Modern Agricultural Industry Technology System (HBCT2018060205).

Institutional Review Board Statement: Not applicable.

Informed Consent Statement: Not applicable.

Data Availability Statement: The data presented in this study are available on request from the corresponding authors.

Conflicts of Interest: The authors declare no conflict of interest.

Sample Availability: Samples of the compounds including protodioscin, protogracillin, pseudoprotodioscin and pseudoprotogracillin are available from the corresponding authors.

\section{References}

1. Ou-Yang, S.H.; Jiang, T.; Zhu, L.; Yi, T. Dioscorea nipponica Makino: A systematic review on its ethnobotany, phytochemical and pharmacological profiles. Chem. Central J. 2018, 12, 1-18. [CrossRef]

2. Zhou, Q.; Sun, H.J.; Liu, S.M.; Jiang, X.H.; Wang, Q.Y.; Zhang, S.; Yu, D.H. Anti-inflammation effects of the total saponin fraction from Dioscorea nipponica Makino on rats with gouty arthritis by influencing MAPK signalling pathway. BMC Complement. Med. Ther. 2020, 20, 1-13. [CrossRef]

3. Hwang, J.T.; Park, K.S.; Ryuk, J.A.; Kim, H.J.; Ko, B.S. Development of an Oriental Medicine Discrimination Method through Analysis of Steroidal Saponins in Dioscorea nipponica Makino and Their Anti-Osteosarcoma Effects. Molecules 2019, $24,4022$. [CrossRef]

4. Liang, X.J.; Guo, Y.C.; Sun, T.Y.; Song, H.R.; Gao, Y.X. Anti-angiogenic effect of total saponins of Rhizoma Dioscorea nipponica on collagen induced-arthritis in rats. Exp. Ther. Med. 2016, 12, 2155-2160. [CrossRef]

5. Zhou, Q.; Yu, D.H.; Zhang, C.; Liu, S.M.; Lu, F. Total saponins from Dioscorea nipponica ameliorate urate excretion in hyperuricemic mice. Planta Med. 2014, 80, 1259-1268.

6. Chien, M.H.; Ying, T.H.; Hsieh, Y.S.; Chang, Y.C.; Yeh, C.M.; Ko, J.L.; Lee, W.S.; Chang, J.H.; Yang, S.F. Dioscorea nipponica Makino inhibits migration and invasion of human oral cancer HSC-3 cells by transcriptional inhibition of matrix metallopro-teinase-2 through modulation of CREB and AP-1 activity. Food Chem. Toxicol. 2012, 50, 558-566. [CrossRef]

7. Li, X.; Wang, Y.; Sun, J.; Li, X.; Zhao, C.; Zhao, P.; Man, S.; Gao, W. Chemotaxonomic studies of 12 Dioscorea species from China by UHPLC-QTOF-MS/MS analysis. Phytochem. Anal. 2020, 31, 164-182. [CrossRef]

8. Guo, L.; Zeng, S.L.; Zhang, Y.; Li, P.; Liu, E.H. Comparative analysis of steroidal saponins in four Dioscoreae herbs by high performance liquid chromatography coupled with mass spectrometry. J. Pharm. Biomed. Anal. 2016, 117, 91-98. [CrossRef]

9. Tang, Y.; Yi, T.; Chen, H.; Zhao, Z.; Liang, Z.; Chen, H. Quantitative Comparison of Multiple Components in Dioscorea nipponica and D. panthaica by Ultra-High Performance Liquid Chromatography Coupled with Quadrupole Time-of-Flight Mass Spectrometry. Phytochem. Anal. 2013, 24, 413-422. [CrossRef]

10. Wang, B.; Ji, S.; Zhang, H.; Zhao, L.; Lv, L.; Li, Y.; Zhou, G.; Zhang, G. Liquid chromatography tandem mass spectrometry in study of the pharmacokinetics of six steroidal saponins in rats. Steroids 2013, 78, 1164-1170. [CrossRef]

11. Chemat, F.; Vian, M.A.; Ravi, H.K.; Khadhraoui, B.; Hilali, S.; Perino, S.; Tixier, A.S.F. Review of Alternative Solvents for Green Extraction of Food and Natural Products: Panorama, Principles, Applications and Prospects. Molecules 2019, 24, 3007. [CrossRef]

12. Shishov, A.; Bulatov, A.; Locatelli, M.; Carradori, S.; Andruch, V. Application of deep eutectic solvents in analytical chemistry. A review. Microchem. J. 2017, 135, 33-38. [CrossRef]

13. Olivares, B.; Martinez, F.; Rivas, L.; Calderon, C.; Munita, M.J.R.; Campodonico, R.P. A natural deep eutectic solvent formulated to stabilize beta-lactam antibiotics. Sci. Rep. 2018, 8, 14900. [CrossRef]

14. Mulia, K.; Nasikin, M.; Krisanti, E.A.; Zahrina, I. Deacidification of Palm Oil Using Betaine Monohydrate-Carboxylic Acid Deep Eutectic Solvents: Combined Extraction and Simple Solvent Recovery. Processes 2020, 8, 543. [CrossRef]

15. Gómez, E.; Cojocaru, P.; Magagnin, L.; Valles, E. Electrodeposition of Co, Sm and SmCo from a Deep Eutectic Solvent. J. Electroanal. Chem. 2011, 658, 18-24. [CrossRef]

16. Shikov, A.N.; Kosman, V.M.; Flissyuk, E.V.; Smekhova, I.E.; Elameen, A.; Pozharitskaya, O.N. Natural Deep Eutectic Solvents for the Extraction of Phenyletanes and Phenylpropanoids of Rhodiola rosea L. Molecules 2020, 25, 1826. [CrossRef]

17. Maiuolo, L.; Algieri, V.; Olivito, F.; De Nino, A. Recent Developments on 1,3-Dipolar Cycloaddition Reactions by Catalysis in Green Solvents. Catalysts 2020, 10, 65. [CrossRef]

18. Liu, Y.; Friesen, J.B.; McAlpine, J.B.; Lankin, D.C.; Chen, S.N.; Pauli, G.F. Natural Deep Eutectic Solvents: Properties, Applications, and Perspectives. J. Nat. Prod. 2018, 81, 679-690. [CrossRef] 
19. Duan, L.; Dou, L.L.; Guo, L.; Li, P.; Liu, E.H. Comprehensive Evaluation of Deep Eutectic Solvents in Extraction of Bioactive Natural Products. ACS Sustain. Chem. Eng. 2016, 4, 2405-2411. [CrossRef]

20. Velásquez, P.; Bustos, D.; Montenegro, G.; Giordano, A. Ultrasound-Assisted Extraction of Anthocyanins Using Natural Deep Eutectic Solvents and Their Incorporation in Edible Films. Molecules 2021, 26, 984. [CrossRef]

21. Islamčević Razboršek, M.; Ivanović, M.; Krajnc, P.; Kolar, M. Choline Chloride Based Natural Deep Eutectic Solvents as Extraction Media for Extracting Phenolic Compounds from Chokeberry (Aronia melanocarpa). Molecules 2020, 25, 1619. [CrossRef]

22. Dai, Y.; Witkamp, G.J.; Verpoorte, R.; Choi, Y.H. Tailoring properties of natural deep eutectic solvents with water to facilitate their applications. Food Chem. 2015, 187, 14-19. [CrossRef]

23. Hammond, O.S.; Bowron, D.T.; Edler, K.J. The Effect of Water upon Deep Eutectic Solvent Nanostructure: An Unusual Transition from Ionic Mixture to Aqueous Solution. Angew. Chem. Int. Ed. 2017, 56, 9782-9785. [CrossRef]

24. Guo, N.; Kou, P.; Jiang, Y.W.; Wang, L.T.; Niu, L.J.; Liu, Z.M.; Fu, Y.J. Natural deep eutectic solvents couple with integrative extraction technique as an effective approach for mulberry anthocyanin extraction. Food Chem. 2019, 296, 78-85. [CrossRef] [PubMed]

25. Bezerra, M.A.; Santelli, R.E.; Oliveira, E.P.; Villar, L.S.; Escaleira, L.A. Response surface methodology (RSM) as a tool for optimization in analytical chemistry. Talanta 2008, 76, 965-977. [CrossRef] [PubMed]

26. Oroian, M.; Ursachi, F.; Dranca, F. Influence of ultrasonic amplitude, temperature, time and solvent concentration on bioactive compounds extraction from propolis. Ultrason. Sonochem. 2020, 64, 105021. [CrossRef] [PubMed]

27. Oomen, W.W.; Begines, P.; Mustafa, N.R.; Wilson, E.G.; Verpoorte, R.; Choi, Y.H. Natural Deep Eutectic Solvent Extraction of Flavonoids of Scutellaria baicalensis as a Replacement for Conventional Organic Solvents. Molecules 2020, 25, 617. [CrossRef]

28. He, X.; Yang, J.; Huang, Y.; Zhang, Y.; Wan, H.; Li, C. Green and Efficient Ultrasonic-Assisted Extraction of Bioactive Components from Salvia miltiorrhiza by Natural Deep Eutectic Solvents. Molecules 2020, 25, 140. [CrossRef]

29. Ojha, K.S.; Aznar, R.; O’Donnell, C.; Tiwari, B.K. Ultrasound technology for the extraction of biologically active molecules from plant, animal and marine sources. Trends Anal. Chem. 2020, 122, 115663. [CrossRef]

30. Hsieh, Y.H.; Li, Y.; Pan, Z.; Chen, Z.; Lu, J.; Yuan, J.; Zhu, Z.; Zhang, J. Ultrasonication-assisted synthesis of alcohol-based deep eutectic solvents for extraction of active compounds from ginger. Ultrason. Sonochem. 2020, 63, 104915. [CrossRef]

31. Duan, L.; Zhang, W.H.; Zhang, Z.H.; Liu, E.H.; Guo, L. Evaluation of natural deep eutectic solvents for the extraction of bioactive flavone C-glycosides from Flos Trollii. Microchem. J. 2019, 145, 180-186. [CrossRef] 